\title{
Modeling of the Fluid Solid Interaction during Seismic
}

\section{Event}

Jan Vachulka*

Stevenson and Associates, Vejprnicka 56, Pilsen, Czech Republic

\begin{abstract}
The fluid solid interaction belongs to one of the important topics in structural design in nuclear field. In the past decades many researchers such[1-3], [6-8] have dedicated large effort to find especially modal shapes and transient response of the cylindrical fluid filled tanks. Less effort was dedicated to rectangular tanks [3, 4]. The main aim of this paper is to present acceptable method for determining fluid-structure interaction during seismic event using standard finite element code without implemented fluid finite elements. The method is based on assumption that in-vacuum modal shapes of the structure are known and also the modal shapes of the free liquid surface are known. The mode shapes in vacuum are determined by standard finite element code and free surface modes are derived analytically or using boundary element method. The boundary condition on the fluid-structure interface is obtained semi-analytically in form of Fourier or Bessel-Fourier series for simple domains or using boundary element method for complicated fluid domains. The fluid is assumed to be irrotational and incompressible. Applying Galerkin method the system of is obtained. The system of equations is solved in time domain using Newmark integration scheme. The seismic response of the liquid-filled tank is calculated. The calculated example shows very good agreement with the previously published. The method can be used in nuclear technology design.
\end{abstract}

Key words: Interaction, fluid, potential, finite element, boundary element.

\section{Introduction}

The incompressible fluid solid interaction problems can be described using following sets of equations:

- La-Place equation in fluid domain $\Delta \varphi=0$

- Free surface condition of the fluid

$$
g \frac{\partial \varphi}{\partial z}+\frac{\partial^{2} \varphi}{\partial t^{2}}=0
$$

- Fluid-solid interface equations

$$
\operatorname{grad}(\varphi) \cdot \vec{n}=\dot{\vec{u}} \cdot \vec{n}
$$

- Solid domain equations (Elasticity equations)

where $\varphi$ is fluid potential, $\vec{u}$ is solid body displacement, $\vec{n}$ is outer normal of fluid-solid interface

The sets of these equations can be solved using FEM, combination of the FEM and BEM or semianalitical methods in form of infinite series for simple domains can be used.

Corresponding author: Jan Vachulka. E-mail: vachulka@stevenson.cz.

\section{Description of Proposed Method}

The proposed method is based on the assumption, that we do know the solution of the Laplace equation and the boundary conditions will be satisfied approximately using infinite series or BEM. The method is based on original assumption of fluid potential decomposition. The primary unknown is the convective part of the fluid potential and deformations of the walls on the fluid-solid interface. The convective part of the fluid potential satisfies the rigid walls boundary condition and can be obtained in closed form for simple domains or using BEM for complicated domains. The deformation of the solid walls can be expressed as the linear combination of the suitable in-vacuum modes. Hence convective part of potential functions and in vacuum modal shapes are full vector spaces.

Summing up the above assumptions we have:

$$
\begin{gathered}
\varphi=\varphi_{S}+\varphi_{B}+\varphi_{R}, \\
\Delta \varphi=\Delta\left(\varphi_{S}+\varphi_{B}+\varphi_{R}\right)=0
\end{gathered}
$$

where $\varphi_{S}$ is convective part of fluid potential 


$$
\begin{aligned}
& \Delta \varphi_{S}=0 \text { on } \Omega_{F} \\
& \frac{\partial \varphi_{S}}{\partial n}=0 \text { on } \Gamma_{S F}
\end{aligned}
$$

The impulsive part of the potential $\varphi_{B}$ satisfies (4), (5), (6):

$$
\begin{gathered}
\Delta \varphi_{B}=0 \text { on } \Omega_{F} \\
\frac{\partial \varphi_{B}}{\partial n}=\operatorname{grad}\left(\varphi_{B}\right) \cdot \vec{n}=\dot{\vec{u}} \cdot \vec{n} \text { on } \Gamma_{S F}, \\
\quad \text { a } \varphi_{B}=0 \text { on } \Gamma_{L}
\end{gathered}
$$

Rigidly impulsive part of the fluid potential $\varphi_{R}$ satisfies (7), (8), (9):

$$
\begin{gathered}
\Delta \varphi_{R}=0 \text { on } \Omega_{F} \\
\frac{\partial \varphi_{R}}{\partial n}=\operatorname{grad}\left(\varphi_{R}\right) \cdot \stackrel{r}{n}=\stackrel{\stackrel{r}{u}}{u_{g}} \cdot \stackrel{r}{n} \text { on } \Gamma_{S F} \\
\varphi_{R}=0 \text { on } \Gamma_{L}
\end{gathered}
$$

All parts of potential are bound on the free surface $\Gamma_{L}$ by following relationship (10)

$$
\frac{\partial \varphi}{\partial n}+\frac{1}{g} \ddot{\varphi}=\frac{\partial\left(\varphi_{S}+\varphi_{B}+\varphi_{R}\right)}{\partial n}+\frac{1}{g} \ddot{\varphi}_{S}=0(10)
$$

In Eqs. (1)-(10)

$\dot{\vec{u}}$ means the temporal derivative of the solid part displacement, $\dot{\vec{u}}_{g}$ is the velocity of the seismic motion, $\vec{n}$ is the outer normal of fluid-solid interface, and $\Omega_{S}, \Omega_{F}, \Gamma_{S F}, \Gamma_{L}$ are visible in Fig. 1

\subsection{Numerical Approach-Weighted Residual Method}

Applying weighted residuum approach, virtual works principle and relations (1)-(10) we get the equation for fluid domain (11) and solid domain (12).

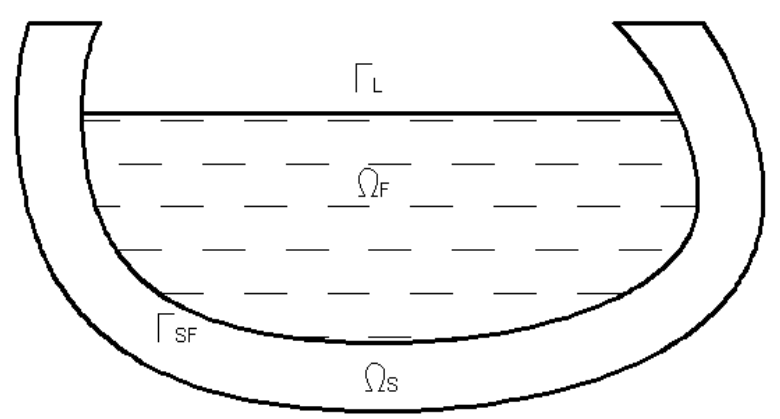

Fig. 1 Fluid and solid domain.

$$
\begin{aligned}
& \int_{\Gamma_{L}} \frac{\partial \varphi_{S}}{\partial n} \delta \varphi_{S} d \Gamma_{L}+ \\
& \int_{\Gamma_{L}} \frac{\partial \varphi_{B}}{\partial n} \delta \varphi_{S} d \Gamma_{L}+\int_{\Gamma_{L}} \frac{\partial \varphi_{R}}{\partial n} \delta \varphi_{S} d \Gamma_{L}+ \\
& \frac{1}{g} \int_{\Gamma_{L}} \ddot{\varphi}_{S} \delta \varphi_{S} d \Gamma_{L}=0 \\
& \int_{\Omega S} \delta \vec{\varepsilon}^{T} \cdot \vec{\sigma} d \Omega_{S}=-\int_{\Gamma_{S F}} \rho_{f} \delta \vec{u}^{T} \vec{n}\left(\dot{\varphi}_{S}+\dot{\varphi}_{B}+\dot{\varphi}_{R}\right) \\
& d \Gamma_{S}-\int_{\Omega_{c}} \delta \vec{u}^{T} \rho_{S}\left(\ddot{\vec{u}}+\ddot{\vec{u}}_{g}\right) d \Omega
\end{aligned}
$$

Introducing the approximations of the solid and fluid region in form:

$$
\begin{gathered}
\vec{u}=\vec{r}_{S}^{T}\left[N_{U}\right] \cdot\{r(t)\} \\
\varphi_{S}=\left[N_{S}\right] \cdot\{s(t)\} \\
\varphi_{B}=\left[N_{B}\right] \cdot\{\dot{r}(t)\} \\
\vec{u}_{g}=u_{g}(t) \cdot \vec{r}_{g} \\
\varphi_{R}=\phi_{R} \cdot \dot{u}_{g}(t) \Rightarrow \frac{\partial \phi_{R}}{\partial n}=\vec{r}_{g} \cdot \vec{n}
\end{gathered}
$$

Substitution into Eqs. (11) and (12) using Green theorem yields in Eq. (18)

$$
\begin{aligned}
& {\left[\begin{array}{cc}
{[\mathrm{K}]} & {[0]} \\
{[0]} & {\left[\mathrm{K}_{\mathrm{F}}\right]}
\end{array}\right] \cdot\left\{\begin{array}{l}
\{\mathrm{r}\} \\
\{\mathrm{s}\}
\end{array}\right\}+\left[\begin{array}{cc}
{[\mathrm{C}]} & {[\mathrm{S}]} \\
{[-\mathrm{S}]^{T}} & {\left[\mathrm{C}_{\mathrm{F}}\right]}
\end{array}\right] \cdot\left\{\begin{array}{l}
\{\dot{\mathrm{r}}\} \\
\{\dot{\mathrm{s}}\}
\end{array}\right\}} \\
& +\left[\begin{array}{cc}
{[\mathrm{M}]+\left[\mathrm{M}_{A}\right]} & {[0]} \\
{[0]} & {\left[\mathrm{M}_{F}\right]}
\end{array}\right] \cdot\left\{\begin{array}{l}
\{\ddot{\mathrm{r}}\} \\
\ddot{\mathrm{s}}\}
\end{array}\right\}=-\left[\begin{array}{ll}
\{L\} & {[0]} \\
{[0]} & \{N\}
\end{array}\right] \cdot\left\{\begin{array}{l}
\ddot{u}_{g} \\
\dot{\mathrm{u}}_{g}
\end{array}\right\}
\end{aligned}
$$

The particles of Eq. (18) are following:

$$
\left[K_{F}\right]=\rho_{f} \cdot \int_{\Gamma_{L}}\left[N_{S}\right]^{T} \rho_{f} \frac{\partial\left[N_{S}\right]}{\partial n} d \Gamma_{L}
$$

which is stiffness matrix of the fluid,

$$
\left[M_{F}\right]=\frac{\rho_{f}}{g} \int_{\Gamma_{L}}\left[N_{S}\right]^{T}\left[N_{S}\right] d \Gamma_{L}
$$

is the mass matrix of the fluid

$$
\{N\}=\rho_{f} \cdot \int_{\Gamma_{L}}\left[N_{S}\right]^{T} \frac{\partial \phi_{R}}{\partial n} d \Gamma_{L}
$$

is the load vector of the fluid 


$$
\begin{gathered}
{[K]=\int_{\Omega_{S}}[B]^{T}[D][B] d \Omega_{S}} \\
\text { is the stiffness matrix of the solid. } \\
{[S]=\rho_{f} \int_{\Gamma_{S F}}\left[N_{U}\right]^{T} \cdot\left\{r_{s}\right\}^{T} \cdot\{n\} \cdot\left[N_{S}\right] d \Gamma_{S F}}
\end{gathered}
$$

is the coupling matrix of the sloshing and bulging part of fluid potential

$$
\left[M_{A}\right]=\rho_{f} \int_{\Gamma_{S F}}\left[N_{U}\right]^{T} \cdot\left\{r_{s}\right\}^{T} \cdot\{n\} \cdot\left[N_{B}\right] d \Gamma_{S F}
$$

is added mass matrix

$$
[M]=\int_{\Omega_{S}}\left[N_{U}\right]^{T} \rho_{S}\left[N_{U}\right] \Omega_{S}
$$

is the mass matrix of the solid

$$
\left\{L_{1}\right\}=\rho_{f} \int_{\Gamma_{S F}}\left[N_{U}\right]^{T} \cdot\left\{r_{s}\right\}^{T} \cdot\{n\} \cdot \phi_{R} \cdot d \Gamma_{S F}
$$

load vector of caused by fluid pressure

$$
\left\{L_{2}\right\}=\rho_{s} \int_{\Omega_{S}}\left[N_{U}\right]^{T}\left\{r_{s}\right\}^{T}\left\{r_{g}\right\} d \Omega_{S}
$$

load vector of caused by solid inertia

$$
\{L\}=\left\{L_{1}\right\}+\left\{L_{2}\right\}
$$

is the total load vector

$[C]$ is damping matrix of the solid

$\left[C_{F}\right]$ is damping matrix of the fluid

\section{Practical Use of the Proposed Method}

If we get input data in the form of the in vacuum modal shapes of the solid part and sloshing potential of the fluid in surrounded by a rigid solid, then applying the boundary conditions (5) and (6) the values of $\varphi_{B}$ can be obtained. Similarly the values of $\varphi_{R}$ can be get from boundary conditions (8) and (9). The input modal shapes can be the functions (especially polynomial) or the nodal displacements (if we use a FEM code). The fluid domain can be simple (rectangular, circular, spherical) and the values for $\varphi_{B}$ and $\varphi_{R}$ can be got in closed form or for complicated domains using boundary integral techniques. The problems lead to system of linear equations if BEM is applied.

The values of $\varphi_{S}$ for rigid solid domains can be got in closed form for simple domains or using boundary integral techniques for complicated domains. The problem leads to eigen-value solution. This results from Eqs. (2), (3) and (10) assume that no seismic motion and rigid walls of the solid part.

Having all the parts of fluid potential and in vacuum modal shapes the fluid-structural matrices can be assembled and seismic FSI problem can be solved.

The method can be used by engineers solving FSI problem having standard FEM code without fluid elements implemented. The method is in fact the Ritz method with in-vacuum modal shapes as base functions of solid and bulging potential and also the sloshing shapes of the fluid in presence of rigid solid as the base functions of the fluid.

\section{The Results and Comparisons}

Previously published seismic response of the tall radial storage tank with rigid bottom was chosen as an example. The radial time histories of the top of the tank were compared. The tank was loaded only in horizontal direction, input motion was EL-Centro. The diameter of tank was $14.604 \mathrm{~m}$, height $21.9456 \mathrm{~m}$, thickness of the shell $25.4 \mathrm{~mm}$. The tank was made of steel and full of the water. Relative damping ratio $2 \%$ was considered. First five members of Fourier-Bessel-series were chosen for approximation of the fluid potentials. The effect of sloshing was not taken into account. The FEM model was constructed using the shell elements in order to calculate in vacuum modal shapes and can be seen on Fig. 2 .

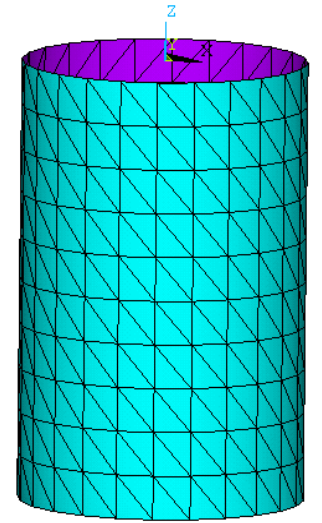

Fig. 2 Finite element model of the storage tank modelled using linear thin-walled shell elements. 

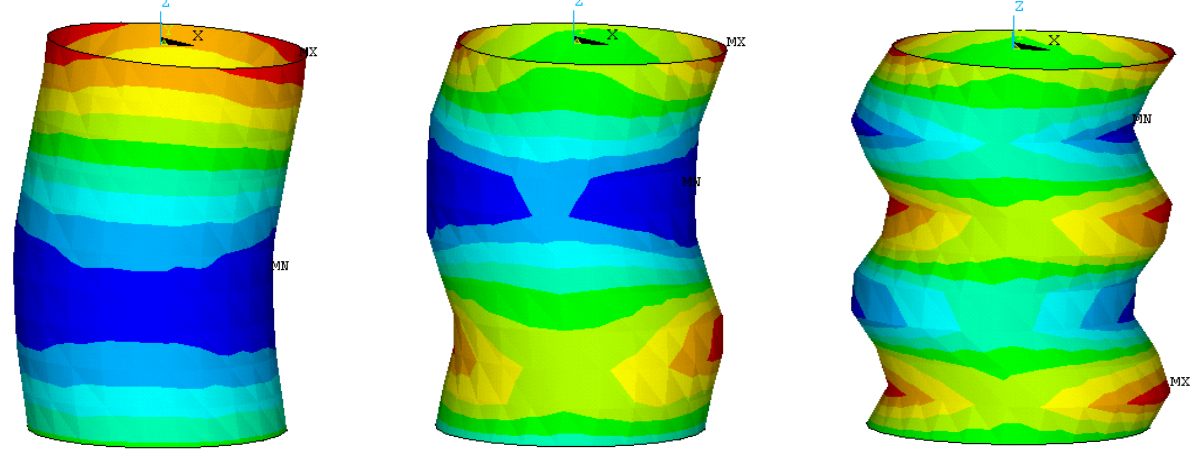

Fig. 3 First three in-vacuum modal shapes used in analysis (frequencies:19.29 Hz, $57.84 \mathrm{~Hz}, 92.59 \mathrm{~Hz}$ ), the total displacements.

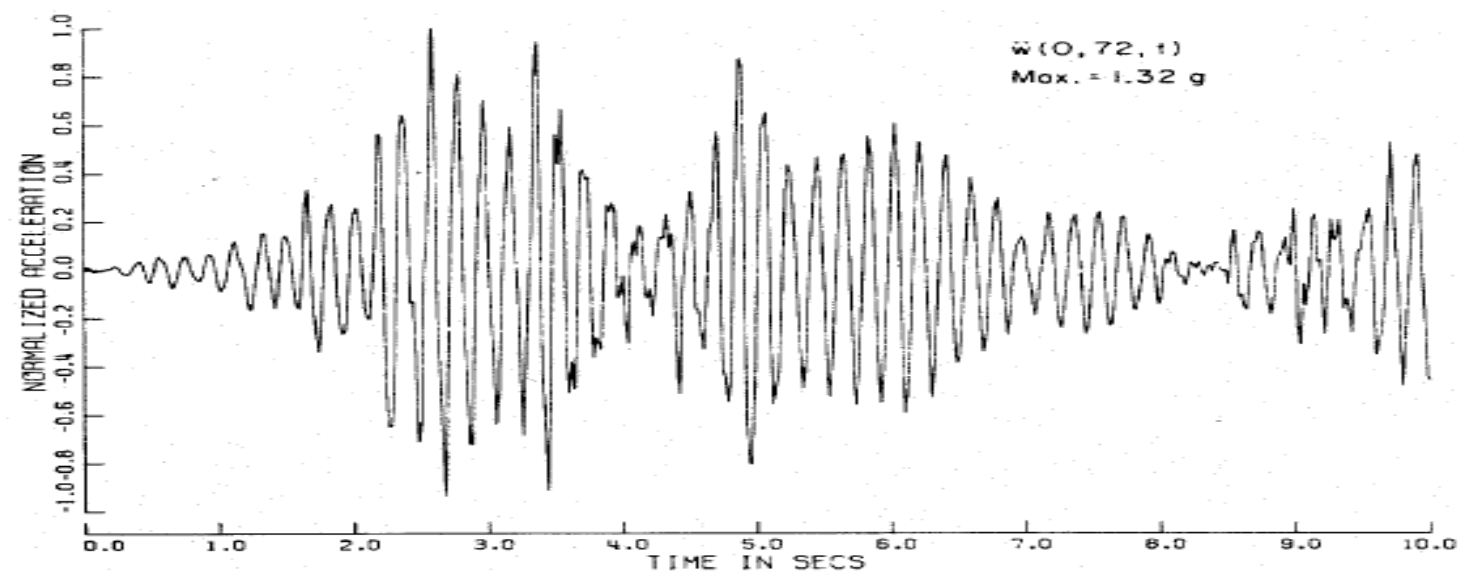

Fig. 4 Time history of acceleration of the top of tall storage tank published by Haroun [3].

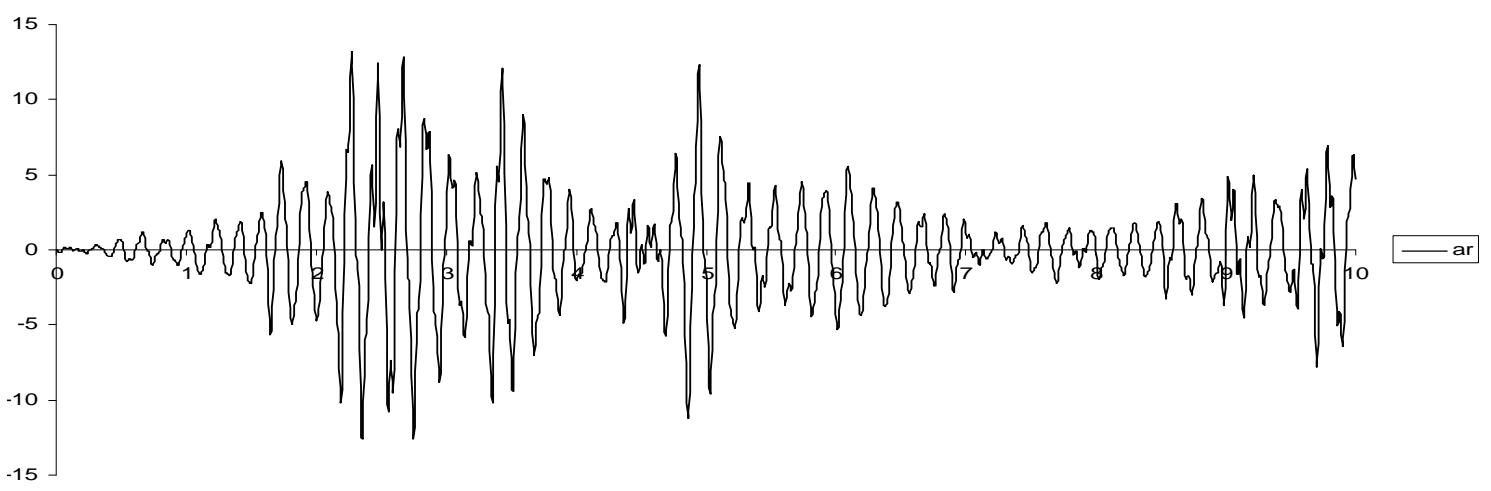

Fig. 5 Time history of acceleration of the top of tall storage tank calculated using proposed method.
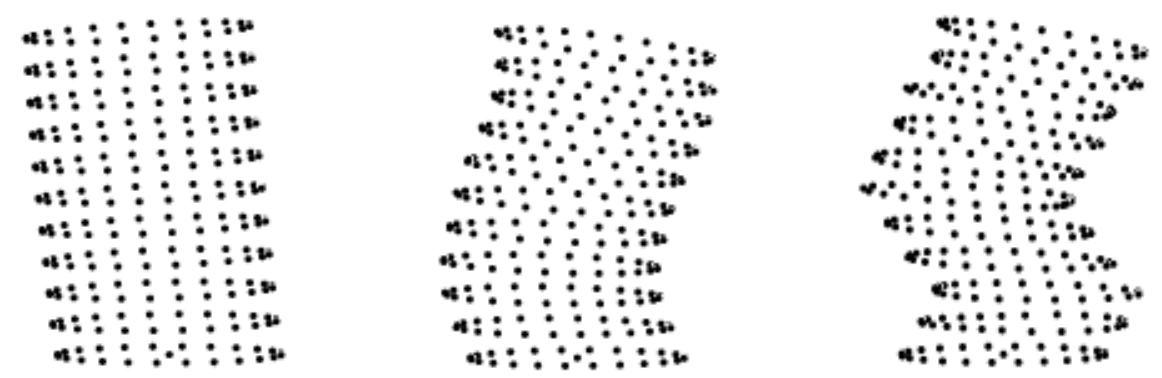

Fig. 6 First three modal shapes of the fluid filled tank (frequencies: $5.40 \mathrm{~Hz}, 16.70 \mathrm{~Hz}, 24.76 \mathrm{~Hz}$ ). 
Table 1 Comparison of the response of the top of the tank.

\begin{tabular}{llll}
\hline Response & $\begin{array}{l}\text { Proposed } \\
\text { method }\end{array}$ & Haroun [3] & $\begin{array}{l}\text { Difference } \\
(\%)\end{array}$ \\
\hline $\begin{array}{l}\text { Radial displacement } \\
(\mathrm{mm})\end{array}$ & 11 & 11.30 & 2.7 \\
$\begin{array}{l}\text { Radial acceleration } \\
\left(\mathrm{ms}^{-2}\right)\end{array}$ & 13.19 & 12.95 & 1.8 \\
\hline
\end{tabular}

Table 2 Table comparison of fundamental frequencies.

\begin{tabular}{lccl}
\hline Frequency & Proposed method & Haroun [3] & $\begin{array}{l}\text { Difference } \\
(\%)\end{array}$ \\
\hline 1 & 5.40 & 5.31 & 2 \\
2 & 16.70 & 15.64 & 6.7 \\
3 & 24.76 & 23.24 & 6.5 \\
\hline
\end{tabular}

\section{Conclusions}

The proposed method showed good agreement with previously published results. The difference in fundamental frequencies and selected responses is acceptable in technical calculations. The difference is mainly caused by the fact that Haroun (1980) had used the closed form solutions for circular shells, but the proposed method uses modes calculated by finite element method. The proposed method can be used by engineers who have to solve the fluid structure interaction problems using standard commercial software without implemented fluid finite elements. This procedure can be also used for solving of dynamic response of the immersed structures in marine engineering. The limitation of proposed method is actually to linear problems, nevertheless the linear approximation is acceptable in many technical topics.

\section{References}

[1] Amabili, M., Paidoussis, M. P., and Lakis, A. A. 1998. "Vibrations of Partially Filled Circular Tanks with Ring Stiffeners and Flexible Bottom.” Journal of Sound and Vibration 213: 259-299.

[2] Amabili, M. 1996. "Free Vibrations of Partially Filled Horizontal Cylindrical Shells.” Journal of Sound and Vibration 191: 757-780.

[3] Haroun, M. A. 1980. Dynamic Analysis of Liquid Storage Tanks. Pasaneda California, 1980.

[4] Jeonga, K. H., Yoo, G. H., and Lee, S. C. 2004. "Hydroelastic Vibration of Two Identical Rectangular Plates.” Journal of Sound and Vibration 272: 539-555.

[5] Zhou, D., and Chang, Y. K. 2000. "Vibration of Vertical Rectangular Plate in Contact with Water on One Side." Earthquake Engineering and Structural Dynamics 29: 693-710.

[6] Goncalves, P. B., and Ramos, N. R. S. S. 1996. "Free Vibrations Analysis of Cylindrical Takes Partially Filled with Liquid." Journal of Sound and Vibration 195: 429-444.

[7] Gupta, R. K. 1995. "Free Vibration of Partially Filled Cylindrical Tanks.” Engineering Structures 17: 221-230.

[8] Gupta, R. K., and Hutchinson, G. L. 1991. "Effect of Wall Flexibility on the Dynamic Response of Liquid Storage Tanks.” Engineering Structures 13: 253-267. 